\title{
Anti-Fibrotic Potential of All Trans Retinoic Acid in Inflammatory Bowel Disease
}

\author{
Dominick L Auci ${ }^{*}$, Nejat K Egilmez ${ }^{2}$ and Gerald W Dryden ${ }^{3}$ \\ ${ }^{1}$ Research and Development TherapyX, Buffalo, NY, USA \\ ${ }^{2}$ University of Louisville, Department of Microbiology and Immunology, Louisville, KY, USA \\ ${ }^{3}$ University of Louisville, Division of Gastroenterology, Hepatology, Nutrition Louisville, KY, USA
}

\begin{abstract}
Received: April 27, 2018; Accepted: May 16, 2018; Published: May 28, 2018
*Corresponding author: Dominick L Auci, Vice President, Research and Development Therapy X, Inc. 138 Farber Hall, 3435 Main Street, Buffalo, NY 14214, USA, Tel: 716-829-2528, E-mail: dauci@therapyXinc.com
\end{abstract}

\section{Introduction}

Inflammatory bowel disease (IBD) embraces Crohn's disease (CD), ulcerative colitis (UC) and the less common indeterminate colitis, all chronic inflammatory processes of the gastrointestinal (GI) tract. Together, they cause significant morbidity for a million and a half Americans [1]. Symptoms include diarrhea, nausea, abdominal pain, weight loss, which can occasionally prove fatal (i.e., toxic megacolon, perforated bowel) [2]. Patients are also at increased risk for colorectal cancer [3]. First line treatments, including various anti-inflammatory agents and antibiotics, are variably effective against active disease [4]. Remissions can be short-lived and uncontrolled activity is associated with significant side-effects [3]. Gut fibrosis will require most patients ( $80 \%$ and $45 \%$ of CD and UC patients, respectively) to undergo surgery [4]. Over the last decade, the so-called biological response modifiers or 'biologics', macromolecules that target inflammatory lymphocytes or the cytokines they produce, have emerged as effective therapeutic agents [5]. For example, infliximab, a chimeric anti-human tumor necrosis factor (TNF)- $\alpha$ antibody earned FDA approval almost 20 years ago, based on a high response rate, significant mucosal and fistula healing and long-term remissions in both CD and UC. Additional targets of biologics, either marketed or in various stages of development, include anti-p40, anti-p19, anti-interleukin (IL)-12/23, and antialpha 4/beta 7 integrin antibodies [5, 6]. However, an estimated $30 \%$ of patients will not respond to such treatments, and of those who initially respond, $50 \%$ will relapse within a year risking significant, often serious side effects including infections and increased risk of cancer. So far, biologics have had only a modest impact on surgery rates [7]. The need for novel therapies remains acute.

Literature reports indicate that All trans retinoic acid (ATRA) provides benefit in various rodent models of IBD, including potential anti-fibrotic activity $[8,9]$. Retinoic acid can exist in the body as any of five different chemical isomers (all-transRA, 9-cisRA, 13-cisRA, 11-cisRA and 9, 13-dicisRA). ATRA is the biologically active isomer and the primary enzymatic product of retinaldehyde oxidation. The retinoic acid family regulates a wide range of biological processes, including embryonic development, reproduction, vision, cell growth and differentiation, as well as, apoptosis and inflammation [10]. Topical ATRA is known as tretinoin, and is currently marketed under several trade names such as Retin- $A \AA$, Retin- $A$ Micro $\AA$, Atralin $\AA$, Renova $\AA$, and Avita $\AA$ as FDA approved topical treatment for acne and skin wrinkles. Oral formulations, called isotretinoin (Accutane $₫$, Claravis $\AA$, Sotret $\AA$ and Amnesteem $₫$ and Vesanoid $囚$ ) can cure severe acne and treat acute promyelocytic leukemia (APL), but oral dosing induces more severe side effects. Isotretinoin has not been tested in IBD clinical trials, perhaps due to the serious side effects associated with oral administration, including teratogenicity, suicidality, headache, dizziness, fever, weakness, tiredness, dry mouth, dry skin, other skin changes, thinning hair, nausea, vomiting, itching, bone pain, mouth sores, increased sweating, earaches, and perhaps even colitis. Black-box warnings for oral formulations have been issued by the FDA because of these serious side effects, including retinoic acid syndrome in APL patients. Retinoic acid syndrome may manifest as life threatening upper respiratory tract disturbances (dyspnea, respiratory insufficiency, pleural effusion, expiratory wheezing and increased susceptibility to pneumonia). Its frequency, mechanism and treatment have recently been reviewed [11]. Efforts to circumvent toxicities by tissue targeted administration have included encapsulation in liposomes and aerosolization, although none of these have progressed further in development or resulted in marketed products $[12,13]$.

This review begins with an analysis of published studies bearing on the efficacy of ATRA as an anti-inflammatory treatment for IBD, including in vitro and in vivo work in rodents, as well as ex vivo studies using human tissues. Clinical reports about ATRA attenuation or induction of IBD will also be reviewed. Efforts to deliver ATRA directly to gut tissue to improve activity and reduce side effects will then be discussed. Finally, the potential of ATRA to treat fibrosis will be considered. If successful, oral delivery of ATRA directly to the immune structures of the gut could represent a novel IBD therapy with a potential to reduce fibrosis and the subsequent need for surgery. 


\section{Efficacy}

\section{Chemical colitis models}

Retinoic Acid has been tested in chemically induced colitis animal models, including murine dextran sulfate sodium (DSS) and 2, 4, 6-trinitrobenzene sulfonic acid (TNBS) models. The TNBS model utilizes $100 \mathrm{mg} / \mathrm{kg}$ TNBS in ethanol administered to rodents intra-rectally. Ethanol disrupts the intestinal barrier and enables the TNBS to haptenize colon tissue proteins. A single TNBS/ethanol challenge leads to an intense immune cell infiltrate into the colonic mucosa and Th1-mediated gut inflammation (see Antoniou et al, for review) [14]. In contrast, the dextran sodium sulfate (DSS) model, the most widely used experimental colitis model, involves administering various concentrations of DSS along with drinking water. Ingestion of DSS over several days results in a bacterial bloom and a subsequent disruption of the intestinal epithelial monolayer lining, allowing entry of enteric contents, including luminal bacteria and their antigens, into the submucosa and underlying tissue. The resultant increases in local cytokines and pro-inflammatory signals (often within 24 hours of challenge) follow a Th2-biased response. The model has been recently and elegantly reviewed by Eichele and Kharbanda [15].

\section{TNBS-induced colitis}

Bai and colleagues, working in Nanchang University in 2009 tested ATRA in the murine TNBS-induced colitis model. In their studies, animals were treated daily (starting 2 hours after TNBS installation) by intraperitoneal injection of $20 \mu \mathrm{g}, 100 \mu \mathrm{g}$, or 300 $\mu \mathrm{g}$ ATRA for 7 days. The group reported dose dependent benefits. High dose ATRA treatment reduced mortality (from 5/18 to $2 / 18$ ) and spared weight loss ( $5 \%$ vs $15 \%$ ). Colon inflammation was reduced, as evidenced by improvements in colon length $(\sim 20 \%)$ and average histological score ( 2.5 vs 3.5$)$ [8]. ATRA treatment significantly reduced tissue levels of myeloperoxidase (MPO) ( 30\%), TNF- $\alpha(\sim 20 \%)$, IL-1 ( 50\%), and IL-6 ( 30\%) compared to untreated controls. Ex vivo results included similar effects on lamina propria mononuclear cells (LPMC) cytokine production. Pro-inflammatory cytokine (TNF- $\alpha$, IL-1, IL-17) production was decreased while regulatory cytokines, such as IL-10 and transforming growth factor (TGF)- $\beta$ were increased compared to untreated animals.

Importantly, ATRA treatment significantly increased (from $2.8 \%+/-0.8 \%$ in controls to $4.6 \%+/-1.1 \%$ in treated animals) LPMC forkhead box (FOXP)3 expression, a marker for regulatory $\mathrm{T}$ cells, while IL-17 expression was concomitantly decreased (from $8 \%+/-1 \%$ to $5.2 \%+/-1.6 \%$ ). With respect to mechanism, animals treated with LE135, an antagonist of retinoic acid receptor (RAR) $\alpha$ experienced opposite effects, and when taken together with the ATRA results, support the hypothesis that during intestinal inflammation, RAR $\alpha$ activation (by ATRA) favors Treg function and inhibits the formation and production of pathogenic Th17cells.

The group augmented their findings in rodents with ex vivo analysis of colonic mucosal biopsies from 10 ulcerative colitis patients and 6 healthy subjects [8]. Tissues were cultured for 18 hours +/-ATRA $(0.1 \mu \mathrm{M})$ or LE135 $(1 \mu \mathrm{M})$. TNF- $\alpha$ levels in supernatants were determination by ELISA and FOXP3 and IL-17 expression quantified by immune histochemistry. Neither FOXP3 nor IL-17 were expressed in healthy colonic tissues; in contrast, a high percentage of LPMC from UC tissues ( 16\%) expressed IL-17, while few LPMC expressed FOXP3 ( 3\%) [8]. When UC specimens were co-cultured with ATRA, FOXP3 expression became detectable in $\sim 7.5 \%$ of LPMCs, while IL-17 expression decreased to detectable in only $\sim 10 \%$ of LPMCs. The addition of LE135, an antagonist of RAR $\alpha$, to culture medium reversed the effects of ATRA. These important observations from human tissue suggest that observations from rodent models may translate directly to human disease.

\section{DSS-induced colitis}

The same group at Nanchang University also tested ATRA in the murine DSS model of acute colitis, using BALB/c mice [9]. After adding $3 \%$ DSS to the drinking water (day 1), either ATRA $(0.5$ $\mathrm{mg}$ ) or the RAR $\alpha$ antagonist LE135 (0.1 mg) was administered by intraperitoneal injection beginning on day 3 . Treatments were repeated daily until the mice were euthanized on day 8. ATRA significantly prevented weight loss compared to LE135 ( 1\% vs $10 \%$ ) and reduced disease activity by $\sim 50 \%$, as demonstrated by longer colon length $(\sim 20 \%)$ and better histological scores $(\sim$ $65 \%$ ). ATRA treatment also significantly reduced levels of MPO $(\sim 25 \%)$ and TNF- $\alpha(\sim 35 \%)$ as well as numbers of colonic CD68 (macrophage marker) and NF-кB positive cells in DSS treated mice compared to untreated controls. Once again, addition of LE135, an antagonist of RAR $\alpha$ produced the opposite effect.

These animal study findings were then confirmed with cell culture. RAW 264.7 cells were pre-treated with vehicle, ATRA $(100 \mathrm{nM})$, or LE135 $(1 \mu \mathrm{M})$ for $1 \mathrm{~h}$, then challenged with LPS (1 $\mu \mathrm{g} / \mathrm{mL}$ ) [9]. Phospho-NF-кB p65 (a marker of activation) was determined by Western blot after one hour. After 24 hours, TNF- $\alpha$ levels were measured in cell supernatants. LPS-stimulated RAW 264.7 cells exhibited early NF-кB p65 phosphorylation, while ATRA exposed cells did not. ATRA suppressed supernatant TNF- $\alpha$ levels by $\sim 40 \%$, while LE135 exhibited opposite effects.

Taken together, these results demonstrate that ATRA improves DSS-induced colitis, in part by decreasing NF- $\kappa B$ activation in gut macrophages.

\section{The TNF $\Delta$ ARE mutation model}

TNF $\Delta$ ARE mice over-produce TNF- $\alpha$ due to deletion of 69 base pairs within the AU-rich element of the TNF gene. This mutation stabilizes TNF mRNA and results in TNF overproduction, leading to the development of chronic ileitis by 20 weeks of age, as first characterized by Collins and colleagues at the University of Colorado in 2011 [16]. This murine model resembles Crohn's disease. A paucity of CD103+ dendritic cells (DC) in draining (mesenteric) lymph nodes and gut lamina propria $(\sim 50 \%$ and $30 \%$, respectively) characterized the chronic ileal inflammation seen at 20 weeks. The importance of this finding relates to the 
fact that CD103+ DCs act as key moderators of ATRA driven tolerance in the gut.

Significant changes in retinaldehyde dehydrogenase (RALDH) levels, the enzymatic machinery responsible for gut ATRA synthesis, were seen, specifically within the DC population [16]. CD103+ DCs, in general, exhibited a three to ten-fold higher expression of RALDH2 mRNA compared to CD103- cells from both wild type and 4-week-old TNFA ARE mice. However, at the 20-week mark, while TNF $\triangle$ ARE mice were in the throes of spontaneous chronic ileal inflammation, their CD103+DC expressed ten-fold lower RALDH2 mRNA levels than wild type CD103+ DCs. Similar observations were made in lamina propria cell RALDH2 and RALDH1. These results suggest that CD103+ DC are the main producers of ATRA in the gut, and that chronic inflammation in the ileum can initiate a down-regulation of ATRA synthesis, coupled with a corresponding reduction intolerogenic DC.

As expected, significant increases in TGF- $\beta(\sim 5$-fold $)$, IL-17A ( $\sim$ 10-fold), IL-6 ( 5-fold) and IL-23 ( 2-fold) mRNA expression accompanied the development of spontaneous ileal inflammation in the LP of 20-week-old TNF $\triangle$ ARE mice as compared to wild type litter mates. A surprising doubling of regulatory $\mathrm{T}$ cells observed in the spleen, lamina propria, and draining mesenteric lymph nodes of inflamed TNF $\triangle$ ARE mice, suggested an alternate source of ATRA in these animals. While exploring for this alternate ATRA source, the researchers found that the RALDH enzymatic machinery in the gut of TNF $\triangle$ ARE mice produced a near doubling of RALDH3 mRNA expression within intestinal epithelial cells.

These observations reveal the interconnection of inflammation and ATRA synthesis. During periods of homeostasis, lamina propria DC cells self-regulate a toleragenic phenotype with intrinsic ATRA synthesis. Either the absence of ATRA or the presence of abundant inflammatory cytokines down regulates RALDH mRNA expression in the CD103+ DC, reducing the number of toleragenic DC. However, intestinal epithelial cells apparently sense local ATRA levels and can up-regulate endogenous ATRA synthesis. This conditional manufacture of ATRA confers the ability to re-establish levels of intestinal ATRA that can be used by regional immune cells to re-establish tolerance, as evidenced by the local and regional Treg expansion. This compensatory effect does not always appear sufficient to abrogate the overwhelming effect of chronic inflammation.

To test the ability of exogenous ATRA to re-establish gut tolerance in the context of disease, 20-week-old TNF $\triangle$ ARE mice were given twice-weekly ATRA injections $(300 \mu \mathrm{g})$ for two weeks [16]. This was followed by histologic and cytometric analysis of immune cells from the spleen, mesenteric lymph nodes and lamina propria. ATRA supplementation significantly decreased markers of both acute (granulocyte infiltration) and chronic (monocytic and lymphocytic infiltration) inflammation by about $50 \%$, compared with vehicle treated controls. Attenuation of established ileitis was closely associated with increasing numbers of functional CD103+ DCs, a modest $(\sim 15 \%)$ but significant increase in regulatory $\mathrm{T}$ cell, and a halving of pathogenic Th17 cell numbers. Cultured lamina propria leukocytes from treated animals showed a highly significant reduction in IL-17 release (70\%), as well as increases in CD103 expression and functional regulatory $\mathrm{T}$ cell numbers compared to findings from the vehicletreated controls.

The important take-away messages from this work include: 1. Compensatory up-regulation of epithelial ATRA synthetic machinery (RALDH3 enzymes) could not re-establish adequate (tolerogenic) concentrations of ATRA in the face of ongoing gut inflammation, possibly because of a progressive loss of intestinal epithelial cell mass; and 2. ATRA supplementation can ameliorate active ileal inflammation, in part by establishing greater numbers of functional CD103+ DCs and regulatory T cells, and a corresponding reduction in lamina propria inhibition Th17 cells and IL-17 production.

\section{ATRA and IBD Correlates in Human IBD Tissues and Controversial Clinical Observations}

\section{ATRA in Human Gut Tissues}

Besides the observations of Bai and colleagues already discussed, additional insight into ATRA effects comes from human tissue studies by Sanders and colleagues, working at the London School of Medicine and Dentistry in 2014 [17]. They investigated potential sources of ATRA in gut tissues from healthy subjects and Crohn's disease (CD) patients. Ileal and colonic tissues were collected from ten CD patients undergoing endoscopy or surgery and compared with similar tissues from healthy subjects. Cell fractions were harvested from these tissue samples and antigen presenting cells (APC) isolated by flow cytometry to assess their RALDH activity. The group first characterized APCs by population markers into myeloid, CD103+, and CD103- dendritic cells. Analysis revealed that the RALDH activity was doubled and tripled within CD103+ and CD103-myeloid dendritic cells (respectively) extracted from CD samples as compared to healthy controls. Although ATRA levels were not measured and the CD sample size was small, the findings indicate that the capacity to generate ATRA is common to multiple APC populations in the human intestine. The upregulation of ATRA generating capacity in CD patients may reflect a compensatory mechanism like the alternate ATRA source found in TNF $\triangle$ ARE mice in the context of chronic intestinal inflammation. Again, despite positive effects on toleragenic CD103+ dendritic cells, RALDH activity appeared insufficient to attenuate disease, or may have even provided ATRA for the differentiation of pro-inflammatory gut macrophages that might have increased intestinal inflammation.

\section{Clinical Controversy}

While the preponderance of in vivo, ex vivo and in vitro evidence from the animal models indicate potential benefits of ATRA supplementation, clinical observations add a layer of complexity to the role of ATRA in intestinal inflammation. Initial clinical observations suggested that ATRA treatments might have triggered cases of new onset UC $[18,19]$. In 2006, Reddy 
and colleagues, working at the University of Chicago, analyzed all reports filed with the Food and Drug Administration (FDA) regarding ATRA and ulcerative colitis. Using the Naranjo adverse drug reaction (ADR) probability scale, they reported that 4 cases (5\%) scored in the "highly probable" range for isotretinoin as the cause of IBD, 58 cases (68\%) were "probable," 23 cases (27\%) were "possible," and no cases were "doubtful" [18]. Since then, several epidemiologic studies from multiple countries have reported conflicting outcomes regarding the association between use of isotretinoin and IBD. Some studies, like the publication from Crockett and colleagues out of the University of North Carolina at Chapel Hill in 2010, reported that ulcerative colitis was strongly associated with prior isotretinoin exposure but found no association between isotretinoin exposure and Crohn's disease. Others, like the 2013 publication of Alhusayna and colleagues from The University of Toronto, found no association, while yet another reported a potential protective effect [20, 21].

Interestingly, in 2013, Stobaugh and colleagues, at the North Shore University Health System of Illinois, analyzed a total of 2,214 IBD cases allegedly resulting from isotretinoin filed with the Food and Drug Administration Adverse Event Reporting System (FAERS) between 2003 and 2011 [22]. Attorneys reported $1944(87.8 \%)$ cases whereas physicians reported 132 (6.0\%) and consumers reported 112 (5.1\%). While report accuracy was not ascertained, the authors noted that for the entire FAERS, only $3.6 \%$ of all reports $(2,451,314)$ for all other drug reactions submitted during the same timeperiod were filed by attorneys. Their analysis indicated a clear distortion of the background reporting rate, and they concluded that the submission of attorney-initiated reports artificially inflated the pharmacovigilance signal of isotretinoinassociated IBD.

This controversy was thoroughly reviewed by Steven S. Coughlin of Emory University in 2015, who concluded that results from the several epidemiologic studies that were completed as of his writing, did not support an association between isotretinoin use and any increased risk of inflammatory bowel disease [23]. No clear evidence of a causal link could be found. Since the Coughlin review, another large meta-analysis, published by Lee and colleagues working at the University of Missouri, also concluded that isotretinoin exposure was associated with neither ulcerative colitis nor Crohn's disease [24].

Most observations from tissue culture, clinical experience and epidemiologic data pertaining to isotretinoin exposure all argue against the likelihood that exogenous ATRA exacerbates or induces IBD. While the absence of harm cannot be seen as a proof of benefit, preclinical work in rodents and one published epidemiological study suggest that ATRA might provide benefit to IBD patients, although none of the clinical studies mentioned were designed to detect a benefit in IBD [21]. The clinical utility of exogenous ATRA as an IBD treatment remains to be tested.

\section{ATRA Potential to Treat IBD}

This begs the question of whether ATRA has the potential to treat IBD. Isotretinoin is prescribed over a wide range of dosages.
Acne is treated with 0.5 to $1.0 \mathrm{mg} / \mathrm{kg} /$ day, given in two divided doses with food for 15 to 20 weeks. Allometric scaling of effective doses $(300 \mu \mathrm{g})$ from rodent IBD models predict that up to 10 fold higher doses could be required for effective treatment of IBD compared to doses given for acne, putting patients at a prohibitively high risk for serious, potentially life-threatening side effects. Since systemic exposure to levels of ATRA predicted to be efficacious for IBD entail unacceptable risks, efforts to translate the immunological benefits of ATRA to IBD patients must focus on formulations such that the active ingredient is delivered directly to the disease microenvironment and/or gut immune structures.

\section{Encapsulation}

One promising approach entails the oral administration of ATRA-loaded micro-particles. Efforts to encapsulate ATRA have included various chitooligosaccharide ride formulations, as well as, poly (lactic-co-glycolic acid) (PLGA) based particles [25, 26]. PLGA encapsulated ATRA particles are of particular interest since they have been tested in vitro and in vivo in rodent IBD models $[27,28]$.

\section{Activity in vitro}

Capurso and colleagues working at Yale University in 2010 reported the development of a PLGA-based particulate drug delivery platform capable of encapsulating and releasing ATRA in vitro [27]. They described a single emulsion nano-particle fabrication technique that produced nano-sized droplets of dichloromethane (DCM) containing dissolved PLGA and ATRA. Evaporation of DCM, washing and lyophilization yielded spherical PLGA particles with ATRA molecules entrapped in the polymer matrix. Observation via electron microscopy revealed smoothsurfaced, spherical particles of roughly similar size $(\sim 0.25 \mu \mathrm{M})$ loaded with $2-3 \mu \mathrm{g} /$ ATRA per mg PLGA. The in vitro ATRA release curve was biphasic, with an initial burst during the first 48 hours followed by slower, steady release over at least 5 days.

The particles were then tested, along with soluble ATRA, for in vitro activity. Th17, regulatory, and naïve $\mathrm{T}$ cells (control) were generated from CD4+ murine splenocyte along with exposure to soluble or particulate ATRA, or to blank (i.e., unloaded) particles. In some experiments, Th17 cells were cultured with 3T3 fibroblasts to mimic the in vivo IL-6 driven positive feedback cycle favoring the development of more Th17 cells. Compared to blank particles, soluble and particulate ATRA abrogated IL17 and IFN $\gamma$ production from Th17 cells. ATRA treatment also decreased intracellular expression of IL-17 (66\%) and the key Th17 transcription factor ROR $\gamma$ (33-50\%). ATRA also enhanced the development of the regulatory $\mathrm{T}$ cell phenotype, both in terms of FOXp3 expression (about 4-fold) and IL-10 production (about 6-fold). Both soluble and particulate ATRA abrogated IL17 dependent IL-6 production by fibroblasts. These effects were generally dose dependent, although the greatest activity was observed at the highest $(10 \mathrm{nM})$ dose. Soluble and particulate ATRA behaved similarly with comparable potency. This work demonstrates that ATRA released from PLGA encapsulation, at 
least in vitro, performs as expected in terms of immunological bioactivity. More specifically, this data suggests that in the context of IBD, ATRA could theoretically provide a benefit, especially if delivered directly to the immune structures of the gut.

\section{Activity in vivo}

Conway and colleagues, working in 2015 at Therapy $X$, a small, Buffalo New York based biotechnology company, reported on the clinical development of very similar PLGA-based particulate ATRA as a potential treatment for IBD [28]. The group is developing a combinatorial particulate product (TreXTAM) containing both the key regulatory cytokine TGF- $\beta$ and ATRA in a 1:2 $w / w$ ratio. In this study, TGF- $\beta$ was encapsulated in poly-lactic acid (PLA) microspheres manufactured using a proprietary encapsulation (EXSTaM) technology [29]. ATRA was encapsulated in poly-lacticco-glycolic acid microspheres manufactured using the solvent evaporation technique [30]. While PLA formulations are better suited to preserve the structural integrity and biological activity of large proteins, both particle types are ideally suited for oral delivery, achieving local, sustained release of drug product to the gut over extended periods [31].

While the Buffalo group reported TreXTAM activity in both the DSS and the SCID mouse adoptive CD4+ CD25- T-cell transfer model of IBD, particulate ATRA was tested only in the transfer model, a model which recapitulates many of the pathological cellmediated immune responses associated with Crohn's disease [28]. The group reported that thrice weekly (for two weeks) oral treatment with $20 \mathrm{mg}$ particles (20 $\mu \mathrm{g}$ ATRA) beginning at disease onset, ameliorated weight loss, significantly reduced histologic evidence of colitis $(\sim 40 \%)$, colon shortening $(\sim 15 \%)$ and dramatically reduced serum amyloid A levels by $75 \%$. While the combination generally performed better, the activity of particulate ATRA alone in this challenging onset model, was still broadly significant.

Importantly, the group also performed initial toxic kinetic studies in rats and found only small amounts of ATRA $\left(\mathrm{C}_{\max } \sim 20\right.$ $\mathrm{ng} / \mathrm{mL}$ ) in the blood within an hour of oral treatment (Figure 1). Levels rapidly declined with $\mathrm{at}_{1 / 2}$ of $143 \mathrm{~min}$ (Table 1). A subsequent GLP 28-day repeat dosing toxicity study in rats indicated a NOAEL greater than doses predicted to be efficacious (data not shown). While these PK results are preliminary, when combined with other observations, they indicate that low systemic ATRA exposure after oral ingestion of particulate material may be related to an initial burst on rehydration (as noted by Capurso and colleagues and confirmed by the group at Buffalo), and that particles remaining in gut tissues release ATRA directly into tissue, slowly, over days, with minimal systemic exposure [27].

The in vitro and in vivo observations with PLGA particulate ATRA, when taken together, suggest that oral treatment can release biologically active ATRA locally, over days, with minimal systemic exposure, obviating ATRA side effects while delivering efficacious drug doses for controlling inflammation in the intestinal tissue and immune structures of the gut. More recently, proprietary spray drying methods have been developed by Therapy $X$ to consistently produce the large quantities of particulate ATRA necessary to conduct clinical studies and potential commercialization. This is a unique accomplishment in the industry.

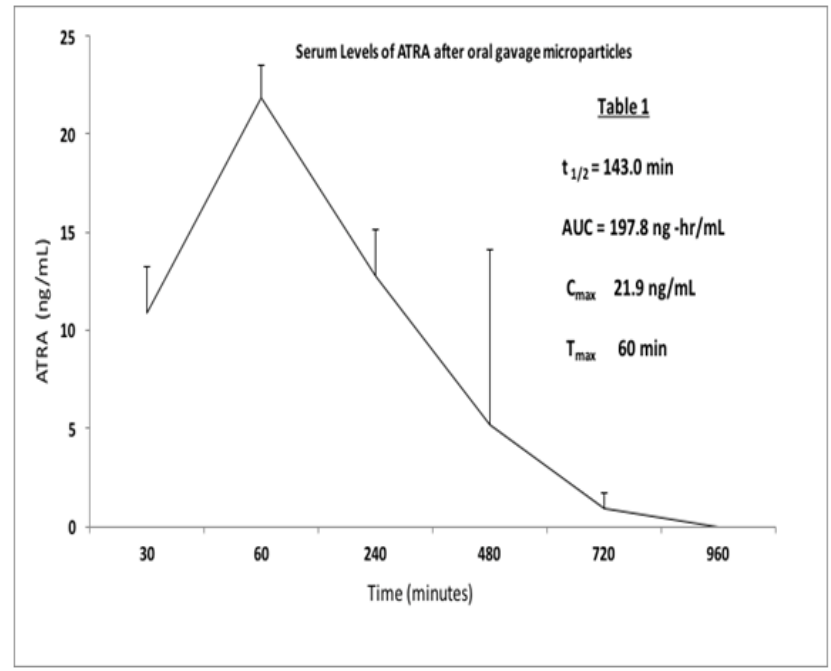

Figure 1: Serum pharmacokinetics of ATRA in naive rats dosed with encapsulated ATRA (18 $\mathbf{~ m g} / \mathbf{k g})$. Sprague-Dawley rats (6 males, 6 females; 12 per group) were dosed $(2 \mathrm{~mL} / \mathrm{kg}$ ) by oral gavage. Animals were bled at each indicated time point after administration of test dose and blood processed to serum. Levels of ATRA were measured by HPLC. Data are expressed as $\mathrm{ng} / \mathrm{mL},+/$ - standard deviation.

\section{ATRA's Potential to Treat Fibrosis}

Intestinal fibrosis remains one of the most serious complications of Crohn's disease, and to a lesser extent, UC. Marked by an excessive deposition of extracellular matrix (ECM) by activated mesenchymal cells, it was thought to be an irreversible, inevitable consequence of chronic inflammation, that, all too often, leads to bowel obstruction and subsequent stricture resection. Because of its alarming contribution to the morbidity and mortality of IBD, intestinal fibrosis has commanded significant attention. Progress on understanding mechanisms, clinical management and potential treatments for IBD fibrosis has been chronicled over the last 20 years in two review articles from the same group, the first published in 2008 and second, more recently in 2017 [32, 33]. Looking back over the last few decades, the group noted that while potent anti-inflammatory biologic therapies have revolutionized IBD treatment, they have made little impact on fibrosis. This not only underscores the urgent unmet medical need for novel therapies, but also challenges the view of inflammation as the sole driver of gut fibrosis and suggests underlying, inflammation-independent self-perpetuating, fibrostenotic processes. Deeper mechanistic understanding should reveal novel targets for therapies specific to type and degree of intestinal fibrosis in IBD. 
While no report establishes the ability of ATRA to treat IBD fibrosis specifically, there is a wide, suggestive literature based on the ability of ATRA to limit fibrosis in various other tissues. However, a small number of reports also suggest that ATRA might enhance ECM production and exacerbate fibrosis both in vitro and in vivo. In 2013, this controversial point was reviewed in detail by Zhou and colleagues [34]. Along with efforts to elucidate mechanism, that body of work strongly suggests that exogenous ATRA treatments could be effective at reducing IBD fibrosis.

\section{Role of ATRA in Organ Fibrosis}

The 70-plus publications reviewed by Zhou from the last 30 years focused on the role of ATRA in liver, lung and kidney fibrosis. In the liver, nine different publications reporting thirteen separate in vivo and in vitro effects of ATRA on fibrotic processes were summarized. Of these, eleven indicated a protective role for ATRA against liver fibrosis/extracellular matrix accumulation while just two, from the same group, suggested a negative role involving the accumulation of ECM in the liver. In lung tissue, five different publications reporting seven separate in vivo or in vitro effects of ATRA on fibrotic processes were summarized, where all seven indicated a protective role for ATRA against pulmonary fibrosis and/or extracellular matrix accumulation. And finally, in kidney tissue, sixteen different publications reporting eighteen separate in vivo or in vitro effects of ATRA on fibrotic processes were summarized, with seventeen indicating a protective role for ATRA against pulmonary fibrosis/extracellular matrix accumulation and only one, a negative role. A summary of mechanistic observations showed that ATRA not only effectively inhibits the expression of various collagens (III, 1A1,Procollagen I) and other pro inflammatory pro-fibrinogenic bio molecules (e.g., $\alpha$-SMA, TNF- $\alpha$, and IL-6 etc), but also fibrosis overall. Therefore, exogenous ATRA would likely play protective roles in fibrotic organ diseases via common pathways.

\section{Mechanisms}

While the anti-inflammatory activity of ATRA in IBD may depend on its ability to attenuate Microbiota induced Th17 driven inflammation via a CD103+ dendritic cell driven expansion of gut regulatory $\mathrm{T}$ cells, a body of evidence also suggests the presence of an inflammation-independent, antifibrotic activity [35]. Gut fibrotic mechanisms are based on the same mechanisms of exaggerated expansion and activation of mesenchymal cells, (fibroblasts, myofibroblasts, and smooth muscle cells) with subsequent accumulation of collagen-rich ECM seen in other organs $[33,36]$. Leakage of enteric flora and other luminal components, immune and non-immune cell products and processes (e.g., proliferation, epithelial and endothelial to mesenchymal and stellate cell transition, migration of bone marrow derived stem cells) combine to drive fibroblast and myofibroblast expansion and activation in parallel with activation of the immune response. Normally, these mechanisms combine to promote a healing response to injury, but in the context of chronic IBD, they drive intestinal fibrosis.

Several studies examining effects of ATRA on the cell types mentioned above support a potential protective effect of ATRA on gut fibrosis. The ability of ATRA to reverse radiation induced fibroblast proliferation in the lung and to limit proliferation and collagen secretion in cardiac fibroblasts was described in $2006[37,38]$. While an older report from 1991 suggested that under inflammatory conditions, ATRA could augment synovial fibroblast proliferation, a recent publication by Shimizu and colleagues reported that ATRA played a key role in reducing liver stellate cell activation [39]. Several additional reports buttress this observation, documenting anti-fibrotic effects on pancreatic stellate cell differentiation and activation [40-43]. Finally, a pair of reports from the early 2000's describes ATRA as a potent inhibitor of both proliferation and migration of human vascular smooth muscle cells and a regulator of ECM turnover [44, 45]. Overall, the activity of ATRA on cells responsible for gut fibrosis would appear to be protective.

\section{Moving Forward}

In addition to inducing and maintaining remission of gut inflammation, preventing or even reversing fibrosis remains the moon shot of IBD therapy [33]. Observations of ATRAinduced anti-inflammatory activity in rodent models suggest a potential role for ATRA in the regression of established fibrotic processes [46]. ATRA's in vitro activity on fibrogenic cells, especially the ATRA-induced quiescence of pancreatic stellate cell differentiation and activation, gains increasing relevance due to its dependence on modulation of the ECM microenvironment [40-43]. The early stages of disease, before ECM deposition and increased stiffness become established may represent the best opportunity for clinical trials in terms of preventing gut fibrosis. However, balancing the serious toxicities associated with systemic administration of efficacious doses means that a shift in clinical equipoise will be required prior to translating the preclinical benefits observed in vitro and in rodent models to IBD patients. The paradigm shift will likely come in the form of novel delivery systems that can deliver and sustain efficacious doses of ATRA to the local, diseased microenvironment, while avoiding toxicities associated with systemic ATRA exposure.

\section{References}

1. Abraham C \& Cho, J.H. Inflammatory bowel disease. The New England journal of medicine. 2009;361:2066-2078. doi: 10.1056/ NEJMra0804647

2. Kraus, S. \& Arber, N. Inflammation and colorectal cancer. Current opinion in pharmacology. 2009;9(4):405-410.

3. Podolsky, D.K. Inflammatory bowel disease. The New England journal of medicine. 2002;347:417-429. doi: 10.1056/NEJMra020831

4. Podolsky, D.K. The current future understanding of inflammatory bowel disease. Best practice \& research Clinical gastroenterology. 2002;16(6):933-943.

5. Rutgeerts P, Vermeire S \& Van Assche G. Biological therapies for inflammatory bowel diseases. Gastroenterology. 2009;136(4):11821197. doi: $10.1053 /$ j.gastro.2009.02.001

6. Neurath M.F. \& Finotto S. Translating inflammatory bowel disease research into clinical medicine. Immunity. 2009;31(3):357-361. 
7. Cannom, R.R., Kaiser, A.M., Ault, G.T., Beart, R.W \& Etzioni, D A. Inflammatory bowel disease in the United States from 1998 to 2005: has infliximab affected surgical rates?. The American surgeon. 2009;75(10):976-980.

8. Bai A1, Lu N, Zeng H, Li Z, Zhou X, Chen J, et al. All-trans retinoic acid ameliorates trinitrobenzene sulfonic acid-induced colitis by shifting Th1 to Th2 profile. Journal of interferon \& cytokine research. 2010;30(6):399-406. doi: 10.1089/jir.2009.0028

9. Hong K1, Zhang Y1, Guo Y2, Xie J3, Wang J1, He X1, Lu N1, Bai A4, et al. All-trans retinoic acid attenuates experimental colitis through inhibition of NF-kappaB signaling. Immunology letters. 2014;162(1):34-40. doi: 10.1016/j.imlet.2014.06.011

10. Stevison, F, Jing J, Tripathy, S \& Isoherranen, N. Role of Retinoic Acid Metabolizing Cytochrome P450s, CYP26, in Inflammation and Cancer. Adv Pharmacol. 2015;74:373-412. doi: 10.1016/ bs.apha.2015.04.006

11. Vahdat L1, Maslak P, Miller WH Jr, Eardley A, Heller G, Scheinberg DA, Warrell RP Jr, et al. Early mortality and the retinoic acid syndrome in acute promyelocy tic leukemia: impact of leukocytosis, low-dose chemotherapy, PMN/RAR-alpha isoform, and CD13 expression in patients treated with all-trans retinoic acid. Blood. 1994 ;84(11):3843-3849.

12.0zpolat B \& Lopez-Berestein G. Liposomal-all-trans-retinoic acid in treatment of acute promyelocy tic leukemia. Leukemia \& lymphoma. 2002;43(5):933-941.

13.Da Li Wang, Melissa Marko, Alan R. Dahl, Kory S. Engelke,1 Michael E. Placke, Anthony R. Imondi, James L. Mulshine, et al. Topical delivery of 13-cis-retinoic acid by inhalation up-regulates expression of rodent lung but not liver retinoic acid receptors. Clinical cancer research: an official journal of the American Association for Cancer Research. 2000;6(9):3636-3645.

14. Efstathios Antoniou, Georgios Antonios Margonis, Anastasios Angelou, Anastasia Pikouli, Paraskevi Argiri, Ioannis Karavokyros, et al. The TNBS-induced colitis animal model: An overview. Ann Med Surg (Lond). 2016; 11: 9-15. doi: 10.1016/j.amsu.2016.07.019

15.Eichele DD \& Kharbanda K.K. Dextran sodium sulfate colitis murine model: An indispensable tool for advancing our understanding of inflammatory bowel diseases pathogenesis. World J Gastroenterol. 2017;23(33):6016-6029. doi: 10.3748/wjg.v23.i33.6016

16. Collins CB, Aherne CM, Kominsky D, McNamee EN, Lebsack MD, Eltzschig $\mathrm{H}$, et al. Retinoic acid attenuates ileitis by restoring the balance between T-helper 17 and $\mathrm{T}$ regulatory cells. Gastroenterology. 2011;141(5):1821-1831. doi: 10.1053/j. gastro.2011.05.049

17. Sanders TJ, McCarthy NE, Giles EM, Davidson KL, Haltalli ML, Hazell S, et al. Increased production of retinoic acid by intestinal macrophages contributes to their inflammatory phenotype in patients with Crohn's disease. Gastroenterology. 2014;146(5):12781288. doi: $10.1053 /$ j.gastro.2014.01.057

18. Reddy D, Siegel CA, Sands BE \& Kane S. Possible association between isotretinoin and inflammatory bowel disease. The American journal of gastroenterology. 2006;101(7):1569-1573.

19. Crockett SD, Porter CQ, Martin CF, Sandler RS \& Kappelman MD.
Isotretinoin use and the risk of inflammatory bowel disease: a case-control study. The American journal of gastroenterology. 2010;105(9):1986-1993. doi: 10.1038/ajg.2010.124

20.Alhusayen RO, Juurlink DN, Mamdani MM, Morrow RL, Shear NH, Dormuth $\mathrm{CR}$, et al. Isotretinoin use and the risk of inflammatory bowel disease: a population-based cohort study. The Journal of investigative dermatol. 2013;133(4):907-912. doi: 10.1038/ jid.2012.387

21. Shadi Rashtak MD, Shahryar Khaleghi PharmD, Mark R. Pittelkow MD. Isotretinoin exposure and risk of inflammatory bowel disease. JAMA dermatology. 2014;150(12):1322-1326.

22.Stobaugh DJ, Deepak P \& Ehrenpreis ED. Alleged isotretinoinassociated inflammatory bowel disease: disproportionate reporting by attorneys to the Food and Drug Administration Adverse Event Reporting System. Journal of the American Academy of Dermatology. 2013;69(3):393-398. doi: 10.1016/j.jaad.2013.04.031

23.Coughlin SS. Clarifying the Purported Association between Isotretinoin and Inflammatory Bowel Disease. J Environ Health Sci. 2015;1(2). doi: 10.15436/2378-6841.15.007

24.Lee SY, Jamal MM, Nguyen ET, Bechtold ML \& Nguyen DL. Does exposure to isotretinoin increase the risk for the development of inflammatory bowel disease? A meta-analysis. Eur J Gastroenterol Hepatol. 2016;28(2):210-216. doi: 10.1097/ MEG.0000000000000496

25.Zhang J, Han J, Zhang X, Jiang J, Xu M, Zhang D, Han J,et al. Polymeric nanoparticles based on chitooligosaccharide as drug carriers for co-delivery of all-trans-retinoic acid and paclitaxel. Carbohydr Polym. 2015;129:25-34. doi: 10.1016/j.carbpol.2015.04.036

26.Lee CM, Park JW, Kim J, Kim DW, Jeong HJ, Lee KY, et al. Influence of histidine on the release of all-trans retinoic acid from selfassembled glycol chitosan nanoparticles. Drug Dev Ind Pharm. 2010;36(7):781-786. doi: 10.3109/03639040903514812

27. Capurso NA, Look M, Jeanbart L, Nowyhed H, Abraham C, Craft J, Fahmy TM, et al. Development of a nanoparticulate formulation of retinoic acid that suppresses Th17 cells and upregulates regulatory T cells. Self/nonself. 2010;1(4):335-340. doi: 10.4161/self.1.4.13946

28.Thomas F Conway, Laura Hammer, Stacia Furtado, Edith Mathiowitz, Ferdinando Nicoletti, Katia Mangano, et al. Oral Delivery of Particulate Transforming Growth Factor Beta 1 and AllTrans Retinoic Acid Reduces Gut Inflammation in Murine Models of Inflammatory Bowel Disease. J Crohns Colitis. 2015;9(8):647-658. doi: 10.1093/ecco-jcc/jjv089

29. Mathiowitz E, Jacob JS, Jong YS, Carino GP, Chickering DE, Chaturvedi P, et al. Biologically erodable microspheres as potential oral drug delivery systems. Nature. 1997;386(6623):410-414. doi: 10.1038/386410a0

30.Jeong YI, SongJG, KangSS, Ryu HH, LeeYH, Choi C, etal. Preparation of poly(DL-lactide-co-glycolide) microspheres encapsulating all-trans retinoic acid. International journal of pharmaceutics. 2003;259(1-2):79-91. doi: 10.1016/S0378-5173(03)00207-2

31. Bakhru SH, Furtado S, Morello AP \& Mathiowitz E. Oral delivery of proteins by biodegradable nano particles. Adv Drug Deliv Rev. 2013;65(6):811-821. doi: 10.1016/j.addr.2013.04.006 
32. Rieder F \& Fiocchi C. Intestinal fibrosis in inflammatory bowel disease - Current knowledge and future perspectives. J Crohns Colitis. 2008;2(4):279-290. doi: 10.1016/j.crohns.2008.05.009

33. Rieder F, Fiocchi C \& Rogler G. Mechanisms, Management, and Treatment of Fibrosis in Patients With Inflammatory Bowel Diseases. Gastroenterology. 2017;152(2):340-350. doi: 10.1053/j. gastro.2016.09.047

34.Zhou T.B, Drummen G.P \& Qin Y.H. The controversial role of retinoic acid in fibrotic diseases: analysis of involved signaling pathways. Int J Mol Sci. 2012;14(1):226-243. doi: 10.3390/ijms14010226

35. Bene K, Varga Z, Petrov V.O, Boyko N \& Rajnavolgyi E. Gut Microbiota Species Can Provoke both Inflammatory and Tolerogenic Immune Responses in Human Dendritic Cells Mediated by Retinoic Acid Receptor Alpha Ligation. Front Immunol. 2017;8:427. doi: 10.3389/ fimmu.2017.00427

36. Bettenworth D \& Rieder F. Pathogenesis of Intestinal Fibrosis in Inflammatory Bowel Disease and Perspectives for Therapeutic Implication. Digestive diseases. 2017;35:25-31.

37. Tabata C, Kubo H, Tabata R, Wada M, Sakuma K, Ichikawa M, etal. Alltrans retinoic acid modulates radiation-induced proliferation of lung fibroblasts via IL-6/IL-6R system. American journal of physiology Lung cellular and molecular physiology. 2006;290(3):597-606. doi: 10.1152/ajplung.00282.2005

38. He Y, Huang Y, Zhou L, Lu LM, Zhu YC, Yao T, et al. All-trans retinoic acid inhibited angiotensin II-induced increase in cell growth and collagen secretion of neonatal cardiac fibroblasts. Acta Pharmacol Sin. 2006;27(4):423-429. doi: 10.1111/j.1745-7254.2006.00296.x

39. Gitter B.D \& Koehneke E.M. Retinoic acid potentiates interleukin-1and fibroblast growth factor-induced human synovial fibroblast proliferation. Clinical immunology and Immunopathology. 1991;61(2):191-201.
40.Shimizu H, Tsubota T, Kanki K \& Shiota G. All-trans retinoic acid ameliorates hepatic stellate cell activation via suppression of thioredoxin interacting protein expression. J Cell Physiol. 2018;233(1):607-616. doi: 10.1002/jcp.25921

41.Jaster R, Hilgendorf I, Fitzner B, Brock P, Sparmann G, Emmrich J, Liebe $S$, et al. Regulation of pancreatic stellate cell function in vitro: biological and molecular effects of all-trans retinoic acid. Biochem Pharmacol. 2003;66(4):633-641.

42.Antonios Chronopoulos, Benjamin Robinson, Muge Sarper, Ernesto Cortes, Vera Auernheimer, Dariusz Lachowski, Simon Attwood, et al. ATRA mechanically reprograms pancreatic stellate cells to suppress matrix remodeling and inhibit cancer cell invasion. Nature Communications.2016;7:12630. doi:10.1038/ncomms12630

43. Froeling FE, Feig C, Chelala C, Dobson R, Mein CE, Tuveson DA, et al. Retinoic acid-induced pancreatic stellate cell quiescence reduces paracrine Wnt-beta-catenin signaling to slow tumor progression. Gastroenterology. 2011;141(4):1486-1497. doi: 10.1053/j. gastro.2011.06.047

44.Axel DI, Frigge A, Dittmann J, Runge H, Spyridopoulos I, Riessen $\mathrm{R}$, et al. All-trans retinoic acid regulates proliferation, migration, differentiation, and extracellular matrix turnover of human arterial smooth muscle cells. Cardiovasc Res. 2001;49(4):851-862.

45.Johst U, Betsch A, Wiskirchen J, Schöber W, Vonthein R, Rinkert N, et al. All-trans and 9-cis retinoid acids inhibit proliferation, migration, and synthesis of extracellular matrix of human vascular smooth muscle cells by inducing differentiation in vitro. J Cardiovasc Pharmacol. 2003;41(4):526-535.

46.Kisseleva $T$ \& Brenner D.A. Anti-fibrogenic strategies and the regression of fibrosis. Best practice \& research Clinical gastroenterology. 2011;25(2):305-317. 